\title{
Rectification at Graphene-Semiconductor Interfaces: Zero-Gap Semiconductor-Based Diodes
}

\author{
S. Tongay, ${ }^{1,2,3}$ M. Lemaitre, ${ }^{1}$ X. Miao, ${ }^{2}$ B. Gila, ${ }^{1,3}$ B. R. Appleton, ${ }^{1,3}$ and A. F. Hebard ${ }^{2}$ \\ ${ }^{1}$ Materials Science and Engineering, University of Florida, Gainesville, Florida 32611, USA \\ ${ }^{2}$ Department of Physics, University of Florida, Gainesville, Florida 32611, USA \\ ${ }^{3}$ Nanoscience Institute for Medical and Engineering Technology, University of Florida, \\ Gainesville, Florida 32611, USA \\ (Received 24 May 2011; published 17 January 2012)
}

\begin{abstract}
Using current-voltage $(I-V)$, capacitance-voltage $(C-V)$, and electric-field-modulated Raman measurements, we report on the unique physics and promising technical applications associated with the formation of Schottky barriers at the interface of a one-atom-thick zero-gap semiconductor (graphene) and conventional semiconductors. When chemical-vapor-deposited graphene is transferred onto $n$-type $\mathrm{Si}, \mathrm{GaAs}, 4 \mathrm{H}-\mathrm{SiC}$, and $\mathrm{GaN}$ semiconductor substrates, there is a strong van-der-Waals attraction that is accompanied by charge transfer across the interface and the formation of a rectifying (Schottky) barrier. Thermionic-emission theory in conjunction with the Schottky-Mott model within the context of bond-polarization theory provides a surprisingly good description of the electrical properties. Applications can be made to sensors, where in forward bias there is exponential sensitivity to changes in the Schottky-barrier height due to the presence of absorbates on the graphene, and to analog devices, for which Schottky barriers are integral components. Such applications are promising because of graphene's mechanical stability, its resistance to diffusion, its robustness at high temperatures, and its demonstrated capability to embrace multiple functionalities.
\end{abstract}

DOI: 10.1103/PhysRevX.2.011002

\section{INTRODUCTION}

Single-atom layers of carbon (graphene) have been studied intensively after becoming experimentally accessible with techniques such as mechanical exfoliation [1], thermal decomposition on $\mathrm{SiC}$ substrates [2], and chemical-vapor deposition (CVD) [3,4]. Graphene is a zero-gap semiconductor with an exotic linearly dispersing electronic structure, high optical transparency, exceptional mechanical stability, resilience to high temperatures, and an in-plane conductivity with unusually high mobility [5]. Accordingly, graphene has been proposed as a novel material for incorporation into devices ranging from Schottky light-emitting diodes [6-8] to field-effect transistors (FETs) [9,10]. Although integration of graphene into semiconductor devices is appealing, there is still very little known about the interface physics at graphene/ semiconductor junctions. To this end, graphene/Si junctions showing successful solar-cell operation have been produced by transferring either CVD-prepared [6] or exfoliated [8] graphene sheets onto $\mathrm{Si}$ substrates. The resulting diodes have shown ideality factors (a measure of deviation from thermionic emission) varying from approximately 1.5 [6], which is close to the ideal value of unity, to values in the range of approximately 5-30 on exfoliated graphene [8], implying that additional

Published by the American Physical Society under the terms of the Creative Commons Attribution 3.0 License. Further distribution of this work must maintain attribution to the author(s) and the published article's title, journal citation, and DOI.
Subject Areas: Electronics, Graphene, Semiconductor Physics

nonthermionic current-carrying processes exist at the graphene/Si interface. Although these results are promising, they nevertheless point to the need for additional research on integrating graphene with technologically important semiconductors.

Here, we report rectification (diode) effects at zero-gap-semiconductor/semiconductor (i.e., graphene/ semiconductor) interfaces on a surprisingly wide variety of semiconductors. In addition to current-voltage measurements, we utilize Hall, capacitance-voltage, and electricfield-modulated Raman techniques to gain heretofore unrecognized insights into the unique physics occurring at the graphene/semiconductor interface. We find that, when CVD-prepared graphene sheets are transferred onto $n$-type $\mathrm{Si}, \mathrm{GaAs}, 4 \mathrm{H}-\mathrm{SiC}$, and $\mathrm{GaN}$ semiconductor substrates, equilibration of the Fermi level throughout the system gives rise to a charge transfer between the graphene and the semiconductor, thereby creating strong rectification (called the "Schottky effect") at the interface. We find that graphene's Fermi level $\left(E_{\mathrm{F}}^{\mathrm{gr}}\right)$ is subject to variation during charge transfer across the graphene/semiconductor interface as measured by in-situ Raman-spectroscopy measurements, unlike conventional metal/semiconductor diodes, where the Fermi level $\left(E_{\mathrm{F}}\right)$ of the metal stays constant due to a high density of states at the Fermi level. These variations become particularly pronounced at high reverse-bias voltages when the induced negative charge in the graphene is sufficient to increase $E_{\mathrm{F}}^{\mathrm{gr}}$ and give rise to increased current leakage. Our observations and interpretation based on a modification of thermionic-emission theory not only provide a new understanding for the 
development of high-frequency, high-power, and hightemperature Schottky-based devices, such as metal/ semiconductor field-effect transistors (MESFETs) and high-electron-mobility transistors (HEMTs), but they also allow us to integrate graphene into semiconductor devices while simultaneously preserving the superior properties of the graphene and avoiding chemical-structural modifications to the semiconductor.

\section{EXPERIMENTAL METHODS}

Our diodes are fabricated by transferring large-scale graphene sheets grown by chemical-vapor deposition directly onto the semiconductor under investigation and allowing van-der-Waals attraction to pull the graphene into intimate contact with the semiconductor. Large-area single-layer graphene sheets were synthesized on $\mathrm{Cu}$ foils via a multistep, low-vacuum-CVD process similar to that used in Ref. [11]. A quartz tube furnace operating in CVD mode was loaded with $25-50-\mu \mathrm{m}$-thick $\mathrm{Cu}$ foils (Puratronic, 99.9999\% Cu), evacuated to 4 mTorr and subsequently heated to $500^{\circ} \mathrm{C}$ under a $25-\mathrm{sccm}$ flow of $\mathrm{H}_{2}$ at 325 mTorr. After a 30-minute soak, the temperature was raised to $1025^{\circ} \mathrm{C}$ for 60 minutes to promote $\mathrm{Cu}$ grain growth (to the point at which mean grain size exceeds $5 \mathrm{~mm}^{2}$, determined by optical microscopy). An initial low-density nucleation and slow-growth phase was performed at $1015^{\circ} \mathrm{C}$ for 100 minutes with a mixture of $\mathrm{CH}_{4}$ and $\mathrm{H}_{2}$ at a total pressure of 90 mTorr and flows of $\leq 0.5$ and $2 \mathrm{sccm}$, respectively. Full coverage was achieved by dropping the temperature to $1000^{\circ} \mathrm{C}$ for 10 minutes while increasing the total pressure and methane flow to 900 mTorr and $30 \mathrm{sccm}$, respectively. A 1.5- $\mu$ m-thick film of poly(methyl methacrylate) (PMMA) (MicroChem, $11 \%$ in anisole) was then spincast onto the $\mathrm{Cu}$ foils at $2000 \mathrm{rpm}$ for 60 seconds. The exposed $\mathrm{Cu}$ was etched in an $\mathrm{O}_{2}$ plasma to remove unwanted graphene from the back side of the samples. The PMMA-supported films were then etched overnight in a $0.05 \mathrm{mg} / \mathrm{L}$ solution of $\mathrm{Fe}(\mathrm{III}) \mathrm{NO}_{3}$ (Alfa Aesar) to remove the copper. The graphene/PMMA films were then washed in deionized water, isopropyl alcohol, and buffered oxide etchant for $10 \mathrm{mi}-$ nutes each. After growth and transfer, the graphene films were characterized and identified using a Horiba-Yvon micro-Raman spectrometer with green, red, and UV lasers.

Commercially available semiconducting wafers were purchased from different vendors. $n$-type $\mathrm{Si}$ and $n$-type GaAs samples were doped with $\mathrm{P}\left(2-6 \times 10^{15} \mathrm{~cm}^{-3}\right)$ and $\mathrm{Si}\left(3-6 \times 10^{16} \mathrm{~cm}^{-3}\right)$, respectively. Epilayers of $n$-type $\mathrm{GaN}$ and $n$-type $4 \mathrm{H}-\mathrm{SiC}, 3-6-\mu \mathrm{m}$ thick, were grown on semiinsulating sapphire substrates with $\operatorname{Si}\left(1-3 \times 10^{16} \mathrm{~cm}^{-3}\right)$ and $\mathrm{N}\left(1-3 \times 10^{17} \mathrm{~cm}^{-3}\right)$ dopants. The doping densities $\left(N_{\mathrm{D}}^{\text {Hall }}\right)$ of the semiconducting wafers (Table I) were measured at $300 \mathrm{~K}$ using the Hall bar geometry depicted in Fig. 1(b). During the sample preparation and before the graphene transfer, the wafers were cleaned using typical surface-cleaning techniques. Ohmic contacts to the semiconductors were formed using conventional Ohmic-contact recipes [12-15]. Multilayer Ohmic contacts were thermally grown on the back and front side of the semiconductor and were annealed at high temperatures using rapid thermal annealing. After the Ohmic contact formation, a $0.5-1.0 \mu \mathrm{m}$-thick $\mathrm{SiO}_{x}$ window was grown on various semiconductors using a plasma-enhanced chemical-vapordeposition system, and gold electrodes approximately 500-nm thick were thermally evaporated onto $\mathrm{SiO}_{x}$ windows at $5 \times 10^{-7}$ Torr. The graphene-contacting areas were squares with sides in the range of 500-2000 $\mu \mathrm{m}$. (Application of isopropyl alcohol improves the success rate of the graphene transfer and does not affect the measurements presented here.) After the deposit of the graphene/PMMA films, the samples were placed in an acetone-vapor-rich container for periods ranging from 10 minutes to approximately 10 hours. (The acetone bath allows slow removal of the PMMA films without noticeable deformation of the graphene sheets.)

Before the graphene transfer, there is an open-circuit resistance between the Au contacts and the semiconductor. After the transfer of the PMMA/graphene bilayer, the graphene makes simultaneous connection to the $\mathrm{Au}$

(a)

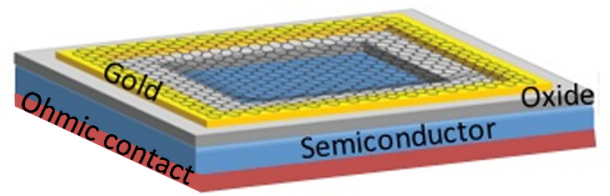

(b)
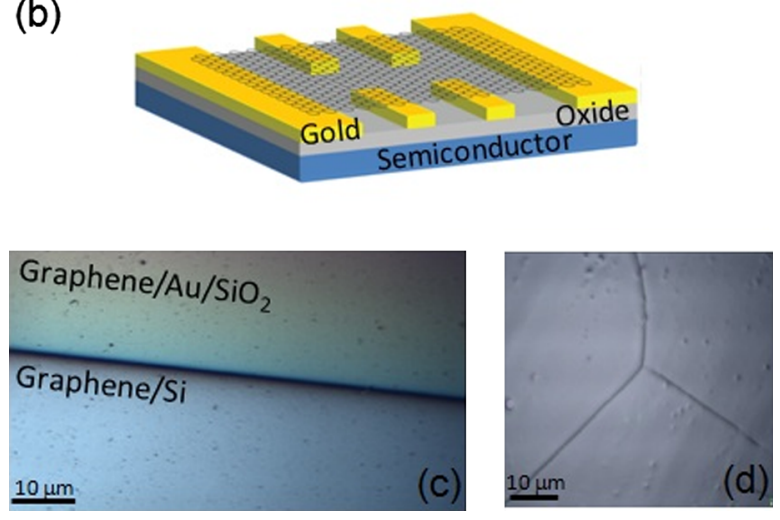

FIG. 1. (a) Graphene/semiconductor diode-sample geometry, where the $J-V$ characteristics are measured between Ohmic contact (ground) and graphene (high). (b) Hall-bar geometry for measurements of the carrier density of graphene. In this configuration, the graphene does not make contact with the semiconductor. (c) Optical image of the graphene/Au/ $\mathrm{SiO}_{2} /$ graphene/Si transition edge after the graphene transfer. (d) Scanning-electron-microscope image of $\mathrm{Cu}$ foils after the graphene growth by CVD, showing the grain boundaries and formation of grain sizes that are large with respect to the $10-\mu \mathrm{m}$ scale bar. 
contacts and the semiconductor, as evidenced by the measured rectifying $I-V$ characteristics. Since the diodes made with the PMMA/graphene bilayer show essentially the same rectifying characteristics as the samples in which the PMMA has been dissolved away, we conclude that the carbon layer on the PMMA (shown by Raman measurements to be graphene) is making intimate contact with the semiconductor.

A schematic for our graphene-based diodes is shown in Fig. 1(a). The back side of the semiconductor substrate is covered with an Ohmic contact, and the graphene sheet is transferred onto $\mathrm{Cr} / \mathrm{Au}$ contacts grown on $\mathrm{SiO}_{x}$ windows. After the transfer, the graphene and semiconductor adhere to each other in an intimate van-der-Waals contact in the middle of the open window, and the $\mathrm{Cr} / \mathrm{Au}$ contact pad provides good electrical contact with the graphene. Our Ohmic-contact arrangements allow current-density-vsvoltage $(J-V)$ and capacitance-vs-voltage $(C-V)$ measurements to be taken separately. $J-V$ measurements were taken in darkroom conditions using a Keithley 6430 subfemptoamp source-meter, and $C-V$ measurements were taken using an HP 4284A capacitance bridge. The electric-fieldmodulated Raman measurements were made on the same configuration. However, four-terminal transport and Hall measurements were performed with an intervening layer of $\mathrm{SiO}_{x}$ [Fig. 1(b)], using a physical-property-measurement system, at room temperature in magnetic fields up to $7 \mathrm{~T}$.

\section{RESULTS AND DISCUSSION}

\section{A. Raman measurements}

In Figs. 2(a)-2(d), we show typical Raman-spectroscopy data taken on graphene sheets grown onto $\mathrm{Cu}$ foils by CVD deposition before and after transferring onto semiconductors. The presented scans have been reproduced at more than 20 random spots and are good representations of the quality of the graphene on the $\mathrm{Cu}$ foils before transfer and on the semiconductor surface after transfer. In the literature, the quality of graphene sheets is measured by a large 2D-to$\mathrm{G}$ intensity ratio $\left(I_{2 D} / I_{G}\right)$ and a low D peak intensity $\left(I_{D}\right)$. Single-layer graphene is expected to show $I_{2 D} / I_{G}>2$, and the amount of disorder in the sheets is often correlated with $I_{D}$. In our samples, we observe $I_{2 D} / I_{G} \geq 2$ and a negligible $D$ peak amplitude. However, after the graphene transfer to the semiconductor substrate, we observe that $I_{D}$ becomes apparent while $I_{2 D} / I_{G}$ remains the same [Fig. 2(b)]. The increase in $I_{D}$ reflects the lower sheet mobility of CVDgrown graphene and gives rise to weak localization effects at low temperatures [16]. Moreover, because of the low solubility of carbon in $\mathrm{Cu}$, graphene growth onto $\mathrm{Cu}$ foils is known to be self-limiting [3], therefore allowing large-area single layers of graphene to be grown onto $\mathrm{Cu}$ foil surfaces. After the graphene growth, the back sides of the $\mathrm{Cu}$ foils were exposed to $\mathrm{O}_{2}$ plasma to remove unwanted graphene and checked with Raman spectroscopy. This step assures

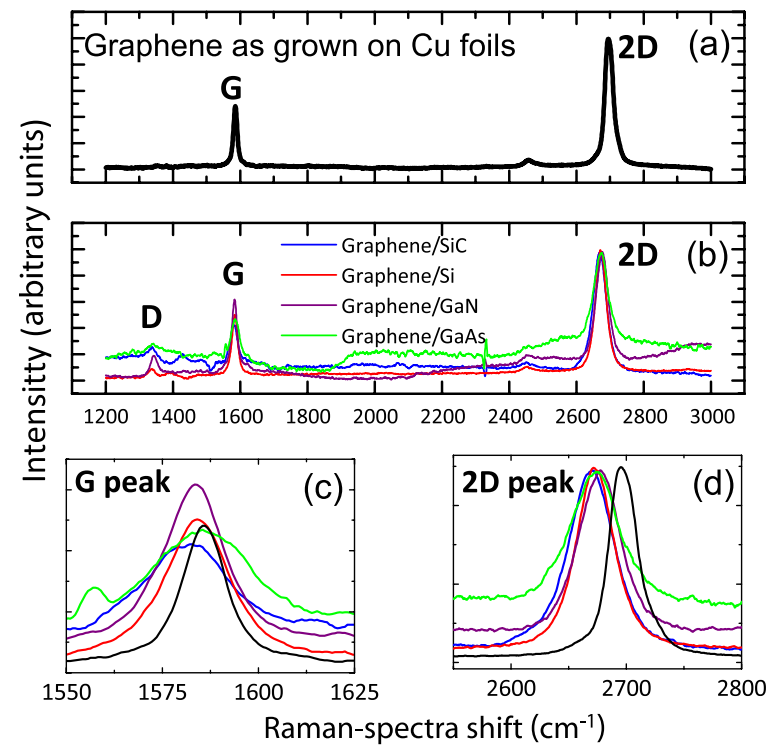

FIG. 2. Raman spectra of (a) CVD-grown graphene on $\mathrm{Cu}$ foils and (b) graphene, after transfer onto various semiconductor substrates. Graphene sheets show a large $I_{2 D} / I_{G}$ ratio, and after the transfer, the graphene becomes slightly disordered. (c) The Raman-spectra $G$ peak. The black curve is the measurement on graphene/Cu and the other curves are for the graphene/semiconductor combinations as indicated in the legend in (b). (d) Same as in (c) for the Raman-spectra $2 D$ peak.

that bilayer (or multilayer) graphene is not formed on PMMA/graphene after etching the $\mathrm{Cu}$ foils. (See Sec. II.)

The Raman spectrum of exfoliated graphene transferred onto $\mathrm{Si} / \mathrm{SiO}_{2}$ substrates has previously been studied as a function of applied bias [17]. It has been found that the G and 2D peaks of graphene are sensitive to the Fermi energy (carrier density) of graphene and allow one to estimate the bias-induced changes in $E_{\mathrm{F}}^{\mathrm{gr}}$. Considering the typical operating voltages of Schottky junctions, the low carrier density in graphene, and the associated bias dependence of $E_{\mathrm{F}}^{\mathrm{gr}}$, we have also measured the Raman spectrum of graphene transferred onto $\mathrm{GaN}$ as a function of applied bias. Our Raman measurements differ from those reported in Ref. [17] in the following three ways: (1) We are using CVD-prepared rather than exfoliated graphene, (2) The graphene is in direct contact with $\mathrm{GaN}$ rather than oxidized $\mathrm{Si}$, and (3) The graphene is measured in situ as part of a Schottky rather than a gated FET. In Fig. 3, we show the evolution of the Raman spectrum as a function of applied bias. While $\mathrm{G}$ and $2 \mathrm{D}$ are almost identical with the same peak positions at $0 \mathrm{~V}$ and $1 \mathrm{~V}$, in reverse bias at $10 \mathrm{~V}$, the $\mathrm{G}$ band shifts higher (by approximately $6 \pm 3 \mathrm{~cm}^{-1}$ ) and the 2D band shifts lower (by approximately $7 \pm 3 \mathrm{~cm}^{-1}$ ) wave numbers. The relative shifts in the Raman peaks along with a reduction of the $2 \mathrm{D} / \mathrm{G}$ peak ratio from 2.6 $($ at $0 \mathrm{~V})$ to $1.2($ at $10 \mathrm{~V})$ imply that graphene sheets transferred onto $\mathrm{GaN}$ become electron doped. Considering the previous results reported on graphene $/ \mathrm{SiO}_{2}[17]$ and doped graphene [18], the shift in $E_{\mathrm{F}}$ can be estimated to be in the range of approximately $0.2-0.5 \mathrm{eV}$. 


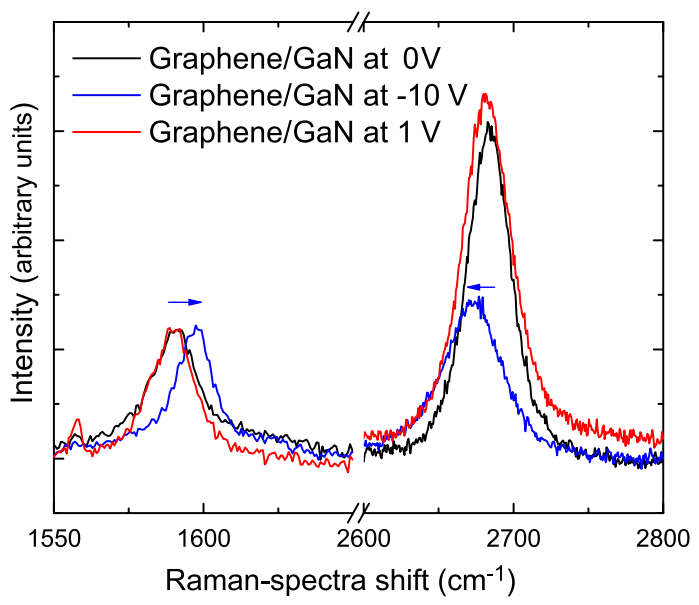

FIG. 3. In-situ Raman spectra taken on graphene/GaN junctions as a function of applied bias: $0 \mathrm{~V}$ (black line), $+1 \mathrm{~V}$ (red line), and $-10 \mathrm{~V}$ (blue line). Blue arrows indicate the direction and magnitude of the shift in the $G$ and $2 D$ peak positions relative to zero bias when the diode is reverse biased.

\section{B. Hall measurements}

Hall measurements show that the Hall mobility of the graphene sheets used in our diodes is in the range of $1400-2100 \mathrm{~cm}^{2} / \mathrm{Vs}$, and that the sheets are hole doped with carrier densities in the range of $2-8 \times 10^{12} \mathrm{~cm}^{-2}$ (Fig. 4). The presence of extrinsic residual doping in exfoliated graphene has been previously reported [1] and attributed to residual water vapor ( $p$ type) or $\mathrm{NH}_{3}$ ( $n$ type). In both cases, annealing reduces the concentration of the dopants and forces $E_{\mathrm{F}}^{\mathrm{gr}}$ closer to the neutrality point. For our CVD-prepared graphene, the presence of residualimpurity doping can be attributed to a lowering of $E_{\mathrm{F}}^{\mathrm{gr}}$ due to hole doping of the graphene during the $\mathrm{Fe}(\mathrm{III}) \mathrm{NO}_{3}$ etching-transfer process [19].

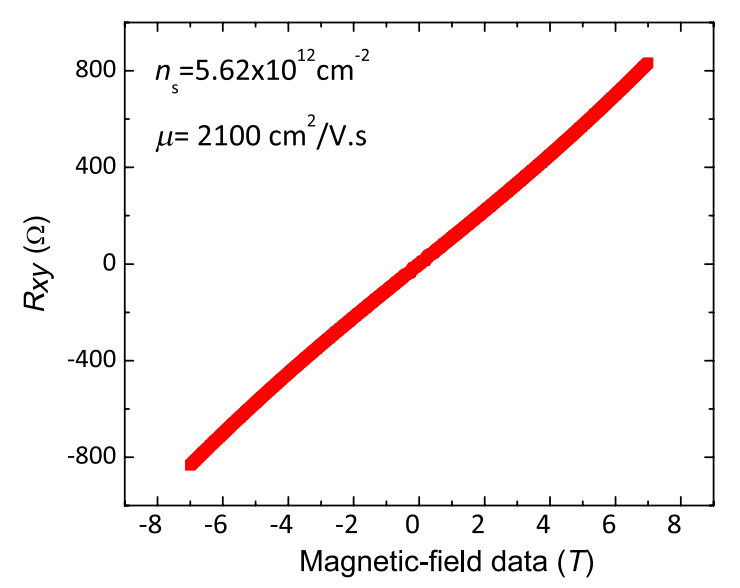

FIG. 4. $R_{x y}$ vs magnetic-field data (T) taken at $300 \mathrm{~K}$. Typically, sample mobilities are in the range of $1400-2100 \mathrm{~cm}^{2} / \mathrm{Vs}$ and carrier densities (holes) are in the range of $2-8 \times 10^{12} \mathrm{~cm}^{2}$.

\section{Current-voltage measurements}

Schottky diodes are expected to pass current in the forward bias (when the semiconductor is negatively biased) while becoming highly resistive in the reverse bias (when the semiconductor is positively biased). As seen in Figs. 5(a)-5(d), $J-V$ (main panels) and $\log J-V$ (insets) data taken on various graphene/ $n$-type semiconductor junctions display strong rectification. This rectification is a consequence of Schottky-barrier formation at the interface when electrons flow from the semiconductor to the graphene as the Fermi energies equilibrate [Fig. 7(b)].
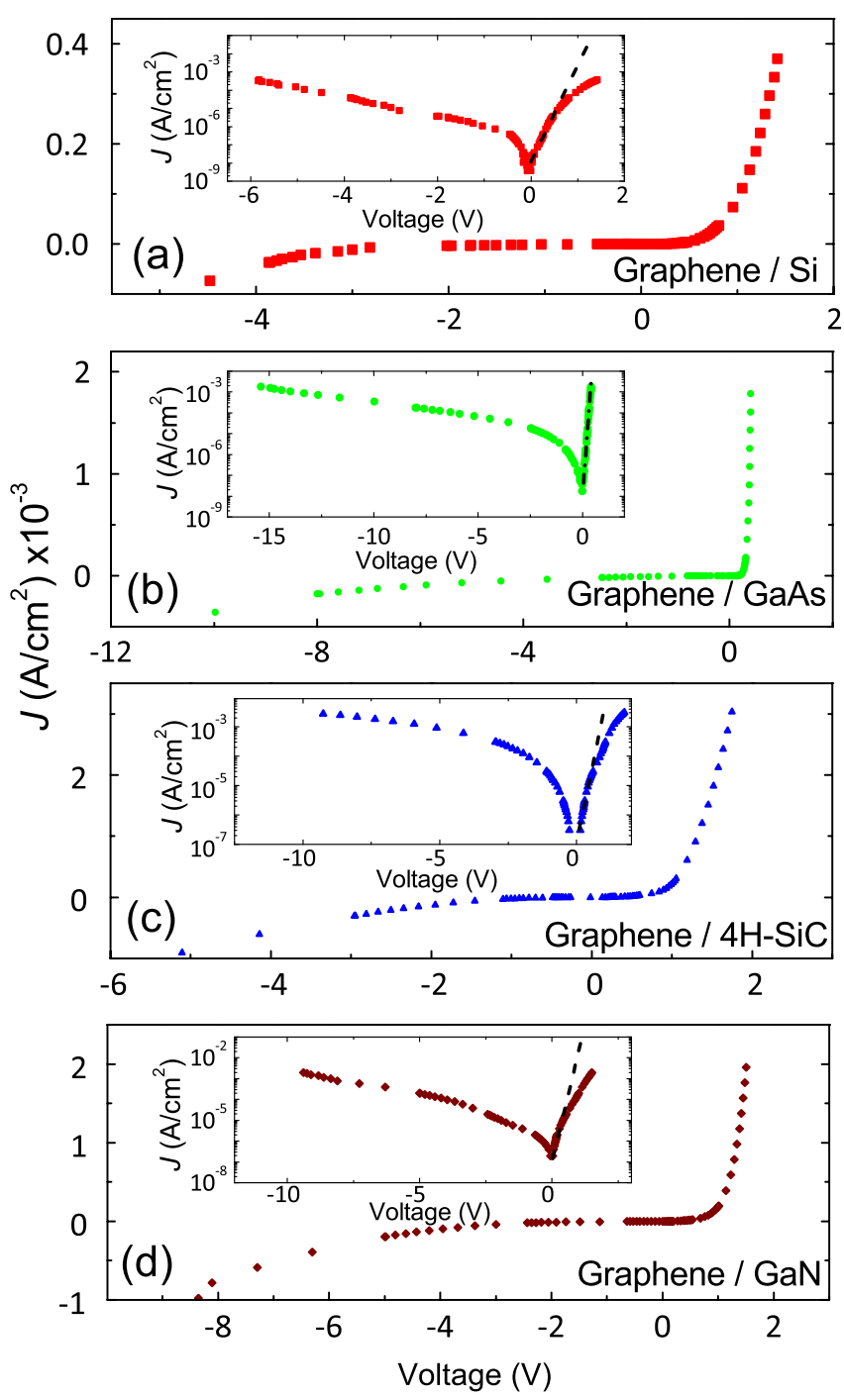

FIG. 5. Room-temperature current-density-voltage characteristics show Schottky rectification at the (a) graphene $/ n-\mathrm{Si}$, (b) graphene $/ n-\mathrm{GaAs}$, (c) graphene $/ n-4 \mathrm{H}-\mathrm{SiC}$, and (d) graphene $/ n$-GaN interfaces. Insets: Semilogarithmic plots, $\log J-V$, reveal a thermionic-emission-dominated current density in forward bias that spans at least two decades of linearity (dashed black lines), allowing us to extract the Schottkybarrier height that is recorded in Table I. The color codes for Fig. 5 are the same as those used for the curves in Fig. 2. 
In principle, any semiconductor with electron affinity $\left(\chi_{\mathrm{e}}\right)$ smaller than the work function of the metal $\left(\Phi_{\text {metal }}\right)$ can create rectification at a metal/semiconductor $(\mathrm{M} / \mathrm{S})$ interface with Schottky-barrier height, $\phi_{\mathrm{SBH}}=$ $\Phi_{\text {metal }}-\chi_{\mathrm{e}}$, given by the Schottky-Mott model. Electron transport over the Schottky barrier at the M/S interface is well described by thermionic-emission theory with the expression

$$
J(T, V)=J_{\mathrm{s}}(T)\left[\exp \left(e V / \eta k_{B} T\right)-1\right],
$$

where $J(T, V)$ is the current density across the graphene/ semiconductor interface, $V$ is the applied voltage, $T$ is the temperature, and $\eta$ is the ideality factor [13]. The prefactor, $J_{\mathrm{S}}(T)$ is the saturation current density and is expressed as $J_{\mathrm{s}}=A^{*} T^{2} \exp \left(-e \phi_{\mathrm{SBH}} / k_{B} T\right)$, where $e \phi_{\mathrm{SBH}}$ is the zero-bias Schottky-barrier height $(\mathrm{SBH})$ and $A^{*}$ is the Richardson constant.

When electronic transport across the barrier is dominated by thermionic emission as described by Eq. (1), semilogarithmic plots of the $J-V$ curves should display a linear region in forward bias. As seen in the insets of Figs. 5(a)-5(d), the overlying dashed straight-line segments of our measurements typically reveal 2-4 decades of linearity, thus allowing us to extract $J_{\mathrm{s}}$ and $\eta$ for each diode. The deviations from linearity at higher bias are due
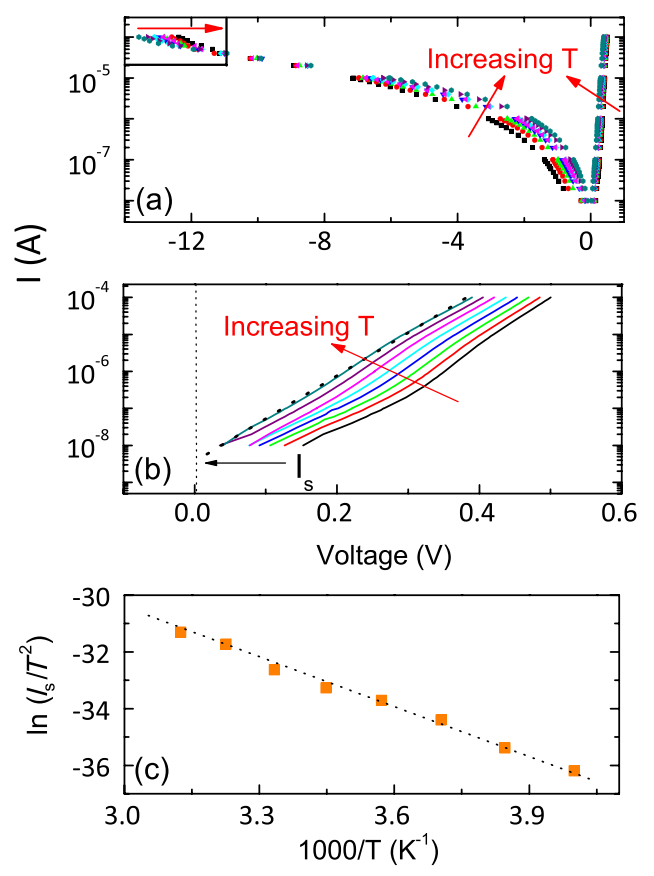

FIG. 6. (a) Plot of the temperature dependence of the current $(I)$ vs voltage $(V)$ curves measured across a graphene/GaAs junction from $250 \mathrm{~K}$ up to $320 \mathrm{~K}$ with $10 \mathrm{~K}$ intervals separating each isotherm. The arrows indicate the direction of increasing temperature. (b) Plot of the temperature dependence of $I-V$ curves taken on graphene/GaAs junctions at different temperatures. (c) Extracted $I_{\mathrm{s}}$ values from Fig. 6(b) are plotted in terms of $\ln I_{\mathrm{s}} / T^{2}$ vs $1000 / T$. to series-resistance contributions from the respective semiconductors. The temperature-dependent data for the graphene/GaAs diode [Figs. 6(a) and 6(b)] show that, for both bias directions, a larger (smaller) current flows as the temperature is increased (decreased), and the probability of conduction electrons overcoming the barrier increases (decreases). In forward bias, the thermionic-emission process manifests itself as linear $\log J-V$ curves [Fig. 6(b)] and linear $\ln \left(I_{s}(T) / T^{2}\right)$-vs- $T^{-1}$ curves [Fig. 6(c)], where $I_{s}(T)=J_{s}(T) A$. The SBH is calculated directly from the slope of this linear dependence. By repeating these temperature-dependent measurements for the four different diodes, we find that the $\mathrm{SBH}\left(\phi_{\mathrm{SBH}}^{J V}\right)$ values at the graphene/semiconductor interfaces are $0.86 \mathrm{eV}, 0.79 \mathrm{eV}$, $0.91 \mathrm{eV}$, and $0.73 \mathrm{eV}$ for $\mathrm{Si}, \mathrm{GaAs}, \mathrm{SiC}$, and $\mathrm{GaN}$, respectively (Table I). While the overall reverse-current density increases as $T$ is increased, we notice that, at high reverse bias, the magnitude of the breakdown voltage $V_{\mathrm{b}}$ decreases linearly with temperature (as shown in the boxed region in the upper left corner of Fig. 6(a)], implying that $V_{\mathrm{b}}$ has a positive breakdown coefficient and that the junctionbreakdown mechanism is mainly avalanche multiplication [13].

The variation of our ideality values in the range of 1.2-5.0 has no obvious correlation to the type of semiconducting substrate. Ideality values greater than one (unity) have been attributed to (1) an image-force-lowering correction to the SBH, (2) a bias-dependent $\mathrm{SBH}$, and (3) the existence of additional current processes such as thermionic-field emission across the metal/semiconductor interface [20]. Here, even though the $E_{\mathrm{F}}^{\mathrm{gr}}$, and hence the SBH of the diode, is bias dependent, we do not expect a large change in the SBH in the forward bias since the applied bias is relatively small. However, even if the current across the $\mathrm{M} / \mathrm{S}$ interface is dominated by thermionic emission, the image-force lowering alone can result in ideality values much larger than unity [20]. Therefore, ideality values exceeding unity might be associated with enhanced image-force lowering across the graphene/ semiconductor interface.

Schottky-barrier values are well described using either the Bardeen or Schottky limits. In the Bardeen limit, the interface physics is mostly governed by interface states which, by accumulating free charge, change the charge

TABLE I. Extracted SBHs, doping densities, and corresponding graphene-work-function values on various graphene/ semiconductor junctions.

\begin{tabular}{lccccc}
\hline \hline Junction type & $\begin{array}{c}\phi_{\text {SBH }}^{J-V} \\
(\mathrm{eV})\end{array}$ & $\begin{array}{c}\phi_{\mathrm{SBH}}^{C-V} \\
(\mathrm{eV})\end{array}$ & $\begin{array}{c}N_{D}^{C-V} \\
\left(\mathrm{~cm}^{-3}\right)\end{array}$ & $\begin{array}{c}N_{D}^{\mathrm{Hall}} \\
\left(\mathrm{cm}^{-3}\right)\end{array}$ & $\begin{array}{c}\Phi_{\mathrm{gr}} \\
(\mathrm{eV})\end{array}$ \\
\hline Graphene $/ n$-Si & 0.86 & 0.92 & $4.0 \times 10^{15}$ & $3.0 \times 10^{15}$ & 4.91 \\
Graphene $/ n$-GaAs & 0.79 & 0.91 & $3.5 \times 10^{16}$ & $3.0 \times 10^{16}$ & 4.89 \\
Graphene $/ n$-4H-SiC & 0.91 & $\cdots$ & $\cdots$ & $1.0 \times 10^{16}$ & 4.31 \\
Graphene $/ n$-GaN & 0.73 & $\cdots$ & $\cdots$ & $1.0 \times 10^{17}$ & 4.83 \\
\hline \hline
\end{tabular}



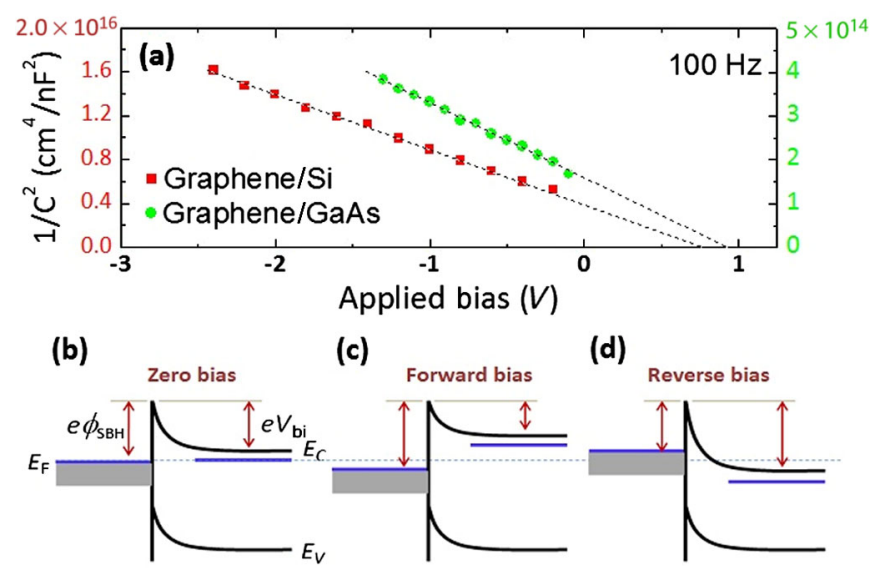

FIG. 7. (a) Plots of the inverse-square capacitance $\left(1 / C^{2}\right)$ vs applied bias $(V)$ for graphene $/ n-\mathrm{Si}$ (red squares and the markings on the left-hand y axis) and for $n$-GaAs (green circles and the markings on the right-hand y axis) at $300 \mathrm{~K}$ and $100 \mathrm{~Hz}$ show a linear dependence, implying that the Schottky-Mott model provides a good description. The interception on the abscissa gives the built-in potential $\left(V_{\mathrm{bi}}\right)$, which can be correlated to the Schottky-barrier height, while the slope of the linear fit gives $2 / e N_{\mathrm{D}} \epsilon_{s} \epsilon_{0}$. Extracted $\phi_{\mathrm{SBH}}$ and $N_{\mathrm{D}}$ values are listed in Table I. Also shown are proposed Schottky-band diagrams of graphene/semiconductor junctions for (b) zero, (c) forward, and (d) reverse bias.

distribution at the interface and cause $E_{\mathrm{F}}$ of the semiconductor to be fixed (Fermi-level pinning). Accordingly, the SBH shows weak dependence on the work function of the metals used for contacts, as found, for example, in GaAs [13]. On the other hand, the wide-band-gap semiconductors $\mathrm{SiC}$ and $\mathrm{GaN}$ are well described by the Schottky-Mott (S-M) limit,

$$
\phi_{\mathrm{SBH}}=\Phi_{\mathrm{gr}}-\chi_{\mathrm{e}}
$$

where $\Phi_{\mathrm{gr}}$ is the work function of the graphene and $\chi$ is the electron affinity of the semiconductor. Using the extracted values of $\phi_{\mathrm{SBH}}$, and electron-affinity values $\left(\chi_{\mathrm{Si}}\right.$ of approximately $4.05 \mathrm{eV}, \chi_{\mathrm{GaAs}}$ of approximately $4.1 \mathrm{eV}$, $\chi_{4 H-\mathrm{SiC}}$ of approximately $3.4 \mathrm{eV}, \quad$ and $\chi_{\mathrm{GaN}}$ of approximately $4.1 \mathrm{eV}$ ), we calculate $\Phi_{\mathrm{gr}}$ (Table I). The calculated values of the work function are typically higher than the accepted values (approximately $4.6 \mathrm{eV}$ ) of graphene when $E_{\mathrm{F}}$ is at the Dirac point (K point). The deviation from this ideal graphene work function can be attributed to the lowering of $E_{\mathrm{F}}$ due to hole doping of the graphene during the $\mathrm{Fe}(\mathrm{III}) \mathrm{NO}_{3}$-etching-transfer process [19] [Figs. 2(c) and 2(d)] together with the fact that the graphene is in physical contact with the gold electrodes [21] (Fig. 4).

Although the SBHs on $\mathrm{Si}, \mathrm{GaAs}$, and $\mathrm{GaN}$ can be roughly explained within the S/M model, in reality, GaAs surfaces have a high density of surface states and thus exhibit characteristic Fermi-level pinning. In the Bardeen limit, GaAs-based diodes generally have SBHs in the range of $0.75-0.85 \mathrm{eV}$ as observed in our measurements, and proper interpretation of the $\mathrm{SBH}$ on GaAs/graphene junctions requires the Bardeen model. After the graphene is placed on the semiconductor surface, there is charge separation and concomitant formation of induced dipoles at the interface. According to bond-polarization theory [20,22], the SBH is determined by charge separation at the boundary between the outermost layers of the metal (here, a single-layer carbon sheet) and the semiconductor. Our results are in good agreement with the findings of our earlier work on graphite and many-layer-graphene junctions where the layer in closest proximity to the semiconductor surface is a single sheet of carbon atoms [23,24]. On the other hand, barriers formed on the $4 \mathrm{H}-\mathrm{SiC}$ substrates give an unphysically low value for $\Phi_{\text {gr }}$ (see Table I) and therefore cannot be explained by either model. The deviation observed on graphene $/ 4 \mathrm{H}-\mathrm{SiC}$ diodes clearly requires consideration of more advanced treatments of metalinduced gap states or bond polarization. For example, since the lattice mismatch between $4 \mathrm{H}-\mathrm{SiC}$ and graphene is quite small when compared to the other substrates (namely, $\mathrm{Si}$, $\mathrm{GaAs}$, and $\mathrm{GaN}$ ), the coupling/interaction between the $4 \mathrm{H}-\mathrm{SiC}$ and graphene might be fundamentally different, and, within the bond-polarization model, this difference might result in the observed deviation.

Next, we turn our attention to reverse-bias characteristics when the semiconductor (graphene) is positively (negatively) charged. In conventional metal/semiconductor Schottky diodes, the work function of the metal is pinned independent of bias voltage due to the high density of states at $E_{\mathrm{F}}$, while, in the reverse (forward) bias, the Fermi energy of the semiconductor shifts down (up), allowing observed rectification via an increase (decrease) in the built-in potential $\left(V_{\mathrm{bi}}\right)$. Unlike conventional metals, graphene's work function $\left(\Phi_{\mathrm{gr}}\right)$ is a function of bias [17], and, for large voltage values, the SBH does not stay constant. When Schottky diodes are forward biased, they pass large currents at voltages well below $1 \mathrm{~V}$, and small decreases in the Fermi level of graphene cannot be distinguished from voltage drops associated with a series resistance. Said in another way, the deviation from linearity in the semilogarithmic plots of Figs. 5(a)-5(d) for forward bias could be due to a combination of a series resistance becoming important at high currents together with a small increase in $\Phi_{\mathrm{gr}}$ and a downward shift in $E_{\mathrm{F}}$ for the positively charged graphene. However, in reverse bias, where the applied voltage can be larger than $10 \mathrm{~V}, E_{\mathrm{F}}$ starts changing dramatically [21] and the fixed-SBH assumption clearly no longer holds. In reverse bias, when the graphene electrodes are negatively charged, $E_{\mathrm{F}}$ increases and $\Phi_{\mathrm{gr}}$ decreases, causing the SBH height to decrease as the reverse bias is increased. As observed in the insets of Figs. 5(a)-5(d), this effect causes the total reverse current to increase as the magnitude of the bias is increased, thus preventing the Schottky diode from reaching 
reverse-current saturation. This nonsaturating reverse current has not been observed in graphite-based Schottky junctions due to the fixed Fermi level of graphite [23].

\section{Capacitance-voltage measurements}

Capacitance-voltage $(C-V)$ measurements made in the reverse-bias mode are complementary to $J$ - $V$ measurements and provide useful information about the distribution and density $N_{\mathrm{D}}$ of ionized donors in the semiconductor and the magnitude of the built-in potential $V_{\mathrm{bi}}$. For a uniform distribution of ionized donors within the depletion width of the semiconductor, the Schottky-Mott relationship between $1 / C^{2}$ and the reverse-bias voltage $V_{\mathrm{R}}$ satisfies the linear relationship, $1 / C^{2}=2\left(V_{\mathrm{R}}+V_{\mathrm{bi}}\right) / e N_{\mathrm{D}} \epsilon_{s} \epsilon_{0}$, which, as shown in Fig. 7(a), is observed to hold for graphene/GaAs and graphene/Si junctions. Linear extrapolation to the intercept with the abscissa gives the built-in potential, $V_{\mathrm{bi}}$, which is related to $\phi_{\mathrm{SBH}}$ via the expression $\phi_{\mathrm{SBH}}=V_{\mathrm{bi}}+$ $e^{-1} k_{b} T \ln \left(N_{\mathrm{c}} / N_{\mathrm{D}}\right)$ [13]. Here, $N_{\mathrm{c}}$ is the effective density of states in the conduction band, $N_{\mathrm{D}}$ is the doping level of the semiconductor, and the slope of the linear fitting to $1 / C^{2}$ vs $V_{\mathrm{R}}$ gives the doping density of the semiconductor. We list $\phi_{\mathrm{SBH}}^{C-V}$ and $N_{\mathrm{D}}$ values for the graphene/GaAs and graphene/ $\mathrm{Si}$ junctions in Table I.

We note from Table I that the extracted $\phi_{\mathrm{SBH}}^{C-V}$ values on the $\mathrm{Si}$ and $\mathrm{GaAs}$ junctions are generally higher than the $\phi_{\text {SBH }}^{J-V}$ values. The discrepancy between the SBHs determined by the two methods can be attributed to: (a) the existence of a thin oxide or residue at the graphene/ semiconductor interface and/or (b) Schottky-barrier inhomogeneity. Graphene sheets transferred onto $\mathrm{SiO}_{2}$ are known to have charge puddles mostly due to the inhomogeneous doping either originating from natural graphite (mechanical-exfoliation transfer) or from chemicals used during the graphene production or transfer (CVD-graphene transfer) process. Since the SBH is sensitive to the $E_{\mathrm{F}}$ of graphene, patches with different charge densities (doping) are expected to have an impact on the SBH and hence on the $J-V$ characteristics of the graphene diodes.

An important difference between the $C-V$ and $J-V$ techniques is that the $C-V$ measurements probe the average junction capacitance at the interface, thereby yielding an average value for the $\mathrm{SBH}$, while the $J-V$ measurements give a minimum value for the $\mathrm{SBH}$, since electrons with thermionic-emission probabilities exponentially sensitive to barrier heights choose low barrier patches (less- $p$-doped graphene patches) over higher patches (more- $p$-doped graphene patches) [20]. While $C-V$ measurements give reasonable values of the $\mathrm{SBH}$ for graphene/GaAs and graphene/Si, we have not been able to obtain reliable $C-V$ measurements for graphene deposited on $\mathrm{GaN}$ and $\mathrm{SiC}$ because of high series resistance in these wide-bandgap semiconductors.

The linearity of the $C$ - $V$ measurements shown in Fig. 7 is consistent with the Schottky-Mott model and the abrupt-junction approximation, which assumes that the density of ionized donors $N_{\mathrm{D}}$ is constant throughout the depletion width of the semiconductor. This good agreement invites a more quantitative analysis of the Fermienergy shifts in the graphene that are the source of the nonsaturating reverse-bias currents discussed in the previous subsection. We begin by writing the electron-charge density per unit area $Q$ on the graphene as

$$
Q=e n_{\text {induced }}=C_{\text {dep }}\left(V_{\mathrm{bi}}+V_{\mathrm{R}}\right),
$$

where

$$
C_{\mathrm{dep}}=\sqrt{\frac{e \epsilon_{s} \epsilon_{0} N_{\mathrm{D}}}{2\left(V_{\mathrm{bi}}+V_{\mathrm{R}}\right)}}
$$

is the Schottky-Mott depletion capacitance, $n_{\text {induced }}$ is the number of electrons per unit area, and $V_{\mathrm{R}}$ is the magnitude of the reverse-bias voltage. Combining these two equations gives the following result:

$$
n_{\text {induced }}=\sqrt{\epsilon_{s} \epsilon_{0} N_{\mathrm{D}}\left(V_{\mathrm{bi}}+V_{\mathrm{R}}\right) / 2 e} .
$$

The above expression provides an estimate of the number of carriers per unit area associated with the electric field within the depletion width, but it does not account for extrinsic residual doping described by the carrier density $n_{0}$ on the graphene before it makes contact with the semiconductor. The processing steps used to transfer the $\mathrm{CVD}$-grown graphene from $\mathrm{Cu}$ substrates to semiconductor surfaces typically results in $p$-doped material with $n_{0}$ of approximately $5 \times 10^{12} \mathrm{~cm}^{-2}$ as inferred from Hall data (Fig. 4) taken at $300 \mathrm{~K}$. Accordingly, the final carrier density including contributions from the as-made graphene and the charge transfers associated with the Schottky barrier $\left(V_{\mathrm{bi}}\right.$ and the applied voltage $\left.V_{\mathrm{R}}\right)$ reads as

$$
n_{\text {final }}=n_{0}-n_{\text {induced }} \text {. }
$$

Using the well-known expression for graphene's Fermi energy [5], we can write

$$
E_{\mathrm{F}}=-\hbar\left|v_{\mathrm{F}}\right| k_{\mathrm{F}}=-\hbar\left|v_{\mathrm{F}}\right| \sqrt{\pi\left(n_{0}-n_{\text {induced }}\right)},
$$

which in combination with Eq. (5) becomes

$$
E_{\mathrm{F}}=-\hbar\left|v_{\mathrm{F}}\right| \sqrt{\pi\left(n_{0}-\sqrt{\epsilon_{s} \epsilon_{0} N_{\mathrm{D}}\left(V_{\mathrm{bi}}+V_{\mathrm{R}}\right) / 2 e}\right)} .
$$

To calculate typical shifts in $E_{\mathrm{F}}$, we use parameter values $\epsilon_{0}=8.84 \times 10^{-14} \mathrm{~F} / \mathrm{cm}^{2}, \hbar=6.5 \times 10^{-16} \mathrm{eVs}$, $e=1.6 \times 10^{-19} \mathrm{C}, \quad v_{\mathrm{F}}=1.1 \times 10^{8} \mathrm{~cm} / \mathrm{s}, \quad V_{\mathrm{bi}} \sim 0.6 \mathrm{~V}$, and $\epsilon_{s}$ of about 10 for a typical semiconductor. Thus the Fermi energy of the as-made graphene with $n_{0}$ of about $5 \times 10^{12} \mathrm{~cm}^{-2}$ is calculated from $E_{\mathrm{F}}=$ $-\hbar\left|v_{\mathrm{F}}\right| \sqrt{\pi n_{0}}$ to be $0.287 \mathrm{eV}$ below the charge-neutrality point, a shift associated with the aforementioned $p$ doping during processing. When the graphene is transferred to the semiconductor, equilibration of the chemical potentials 
and concomitant formation of a Schottky barrier (Fig. 7) result in a transfer of negative charge to the graphene and an increase in $E_{\mathrm{F}}$ [calculated from Eq. (8) for $V_{\mathrm{R}}=0$ ] to be in the range of 3-11 meV for $N_{\mathrm{D}}$ in the range of $1 \times 10^{16}$ to $1 \times 10^{17} \mathrm{~cm}^{-3}$. The application of a typical $10-\mathrm{V}$ reversebias voltage (Figs. 5 and 6) creates significantly larger Fermi-energy shifts, which from Eq. (8) give $E_{\mathrm{F}}$ in the range of $-0.271--0.233 \mathrm{eV}$ for the same factor of 10 variation in $N_{\mathrm{D}}$. The corresponding shifts from the pristine value of $-0.287 \mathrm{eV}$ are in the range of $15-53 \mathrm{meV}$ and thus bring $E_{\mathrm{F}}$ closer to the neutrality point. These numerical calculations show that, for our $n$-doped semiconductors, it is relatively easy to induce Fermi-energy shifts on the order of $50 \mathrm{meV}$ with the application of a sufficiently high reverse-bias voltage. An upward shift in $E_{\mathrm{F}}$ of $50 \mathrm{meV}$ causes a reduction in $\Phi_{\mathrm{gr}}$ by the same amount. Since the electron affinity of the semiconductor remains unchanged, the Schottky-Mott constraint of Eq. (2) enforces the same reduction in $\phi_{\mathrm{SBH}}$, thus leading to a greater than 5\% reduction in the measured SBHs shown in Table I. We note that the induced shift in graphene's $E_{\mathrm{F}}$ as determined by the in-situ Raman-spectroscopy measurements (Fig. 3) is larger ( $\Delta E_{\mathrm{F}}$ of approximately $200-500 \mathrm{meV}$ ) than our theoretical estimation $\left(\Delta E_{\mathrm{F}}\right.$ of approximately $50 \mathrm{meV}$ ).

The discrepancy between the theoretical estimate of $E_{\mathrm{F}}$ and the experimental values might be attributed to (1) the existence of an interface capacitance induced by dipoles at the graphene/semiconductor interface (within bond-polarization theory), causing deviation from the ideal Schottky-Mott capacitance relation given by Eq. (4) and (2) the estimate of $E_{\mathrm{F}}$ using relative peak shifts in the $G$ and $2 D$ peak positions for graphene deposited on $\mathrm{Si} / \mathrm{SiO}_{2}$ [17], which might be different than the change in Fermi level for graphene transferred onto semiconductors.

\section{E. Modification of thermionic-emission theory}

As discussed in the previous sections, since the $E_{\mathrm{F}}$ of the graphene electrode is sensitive to the applied bias across the graphene/semiconductor interface, the SBH at the interface becomes bias dependent, especially for large reverse voltages. However, extracting the SBH from $J$ - $V$ characteristics using Eq. (1), which involves extrapolating current density to zero-bias saturation current $\left(J_{\mathrm{s}}\right)$, yields the putative zero-bias barrier height (Table I). In this section, we present a simple modification to the Richardson equation [Eq. (1)], considering the shift in $E_{\mathrm{F}}$ of graphene induced by applied bias. The modified Richardson equation preserves the original functional form of Eq. (1) but allows one to estimate the SBH at fixed voltages.

The voltage-dependent $\mathrm{SBH}\left[\Phi_{\mathrm{SBH}}(V)\right]$ can be written as

$$
e \Phi_{\mathrm{SBH}}=e \Phi_{\mathrm{SBH}}^{0}+e \Delta \Phi_{\mathrm{SBH}}(V)=e \Phi_{\mathrm{SBH}}^{0}-\Delta E_{\mathrm{F}}(V),
$$

where $e \Phi_{\mathrm{SBH}}^{0}$ is the zero-bias $\mathrm{SBH}$ and $e \Delta \Phi_{\mathrm{SBH}}(V)$ is the correction to the $\mathrm{SBH}$ at fixed voltage $V$. The change in the Fermi energy $\Delta E_{\mathrm{F}}(V)$ is opposite to $e \Delta \Phi_{\mathrm{SBH}}(V)$, i.e., $\Delta E_{\mathrm{F}}(V)=-e \Delta \Phi_{\mathrm{SBH}}(V)$, as seen in Fig. 7(b). Thus, for reverse bias (the addition of electrons to the graphene), we use Eq. (5) in Eq. (7), together with the inequality $n_{\text {induced }} \ll n_{0}$, to calculate

$$
\begin{aligned}
e \Delta \Phi_{\mathrm{SBH}}\left(V_{\mathrm{R}}\right) & =-\Delta E_{\mathrm{F}}\left(V_{\mathrm{R}}\right) \\
& =\hbar v_{F}\left[\sqrt{\pi\left(n_{0}-n_{\text {induced }}\right)}-\sqrt{\pi n_{0}}\right] \\
& \approx-\frac{1}{2} \hbar v_{F} \sqrt{\pi n_{0}} \frac{n_{\text {induced }}}{n_{0}} \\
& =-\frac{1}{2} \hbar v_{\mathrm{F}} \sqrt{\frac{\pi \epsilon_{\mathrm{s}} \epsilon_{0} N_{\mathrm{D}}\left(V_{\mathrm{bi}}+V_{\mathrm{R}}\right)}{2 e n_{0}}} .
\end{aligned}
$$

Adding the reverse and forward current densities, as done in standard treatments of the diode equation [13], yields the total current density across the graphene/semiconductor interface,

$J(V)=A^{*} T^{2} \exp \left(-\frac{e \Phi_{\mathrm{SBH}}^{0}+e \Delta \Phi_{\mathrm{SBH}}(V)}{k_{B} T}\right)\left[\exp \left(\frac{e V}{k_{B} T}\right)-1\right]$.

Here, we note that the original form of the Richardson equation is preserved, with slight modifications to the saturation current term, which is given as

$$
J_{\mathrm{s}}=A^{*} T^{2} \exp \left(-\frac{e \Phi_{\mathrm{SBH}}^{0}+e \Delta \Phi_{\mathrm{SBH}}(V)}{k_{B} T}\right),
$$

with $\Delta \Phi_{\mathrm{SBH}}(V)$ for reverse bias given by Eq. (10).

In our conventional $J-V$ analysis using Eq. (1), the zerobias saturation current $J_{\mathrm{s}}$ is extracted by extrapolating the current density to the zero-bias limit. In this limit, the correction to the SBH is expected to be zero, since the graphene is not subject to applied bias and hence the Fermi level does not shift from the original value. However, using the extrapolated zero-bias saturation-current density, one can extract the SBH, and one can take into account the correction to the $\mathrm{SBH}$ at fixed bias $(V)$ by the additional term $\Delta \Phi_{\mathrm{SBH}}(V)$ in Eq. (12).

\section{CONCLUSION}

In summary, we have used current-voltage and capacitance-voltage measurements to characterize the Schottky barriers formed when graphene, a zero-gap semiconductor, is placed in intimate contact with the $n$-type semiconductors $\mathrm{Si}, \mathrm{GaAs}, \mathrm{GaN}$ and $\mathrm{SiC}$. The good agreement with Schottky-Mott physics within the context of bond-polarization theory is somewhat surprising since the Schottky-Mott picture has been developed for metal/ semiconductor interfaces, not for the single-atomic-layer zero-gap-semiconductor/semiconductor interfaces discussed in this paper. Moreover, due to a low density of 
states, graphene's Fermi level shifts during the charge transfer across the graphene/semiconductor interface. This shift does not occur at metal/semiconductor or graphite/semiconductor interfaces, where $E_{\mathrm{F}}$ remains fixed during Schottky-barrier formation and the concomitant creation of a built-in potential, $V_{\mathrm{bi}}$ with associated band bending. (See Fig. 7.) Another major difference becomes apparent when the diode is under strong reverse bias. According to our in-situ Raman-spectroscopy measurements, large voltages across the graphene/semiconductor interface change the charge density and hence the Fermi level of graphene as determined by relative changes in the $G$ and $2 D$ peak positions. The bias-induced shift in the Fermi energy (and hence the work function) of the graphene causes significant changes in the diode current. Considering changes in the barrier height associated with the bias-induced Fermi-level shift, we modify the thermionic-emission theory, allowing us to estimate the change in the barrier height at fixed applied bias. The rectification effects observed on a wide variety of semiconductors suggest a number of applications, such as to sensors where, in forward bias, there is exponential sensitivity to changes in the SBH due to the presence of absorbates on the graphene, or to MESFET and HEMT devices, for which Schottky barriers are integral components. Graphene is particularly advantageous in such applications because of its mechanical stability, its resistance to diffusion, its robustness at high temperatures, and its demonstrated capability to embrace multiple functionalities.

\section{ACKNOWLEDGMENTS}

We thank Noone Savage for her help during preparation of the diagrams used in this manuscript and Professor A. Rinzler and Dr. M. McCarthy for technical assistance. This work is supported by the Office of Naval Research (ONR) under Contract No. 00075094 (B.R. A.) and by the National Science Foundation (NSF) under Contract No. 1005301 (A.F.H.). Publication of this article was funded in part by the University of Florida Open-Access Publishing Fund.

[1] K. S. Novoselov, A. K. Geim, S. V. Morozov, D. Jiang, Y. Zhang, and S.V. Dubonos, Electric Field Effect in Atomically Thin Carbon Films, Science 306, 666 (2004).

[2] C. Berger, Z. Song, T. Li, X. Li, A. Y. Ogbazghi, R. Feng, Z. Dai, A. N. Marchenkov, E. H. Conrad, P. N. First, and W. A. de Heer, Ultrathin Epitaxial Graphite: 2D Electron Gas Properties and a Route toward GrapheneBased Nanoelectronics, J. Phys. Chem. B 108, 19912 (2004).

[3] X. Li, W. Cai, J. An, S. Kim, J. Nah, D. Yang, R. Piner, A. Velamakanni, I. Jung, E. Tutuc et al, Large-Area
Synthesis of High-Quality and Uniform Graphene Films on Copper Foils, Science 324, 1312 (2009).

[4] K. S. Kim, Y. Zhao, H. Jang, S. Y. Lee, J. M. Kim, K. S. Kim, J. Ahn, P. Kim, J. Choi, and B. H. Hong, Large-Scale Pattern Growth of Graphene Films for Stretchable Transparent Electrodes, Nature (London) 457, 706 (2009).

[5] A.H. CastroNeto, F. Guinea, N. M. R. Peres, K. S. Novoselov, and A. K. Geim, The Electronic Properties of Graphene, Rev. Mod. Phys. 81, 109 (2009).

[6] X. Li, H. Zhu, K. Wang, A. Cao, J. Wei, C. Li, Y. Jia, Z. Li, X. Li, and D. Wu, Graphene-on-Silicon Schottky Junction Solar Cells, Adv. Mater. 22, 2743 (2010).

[7] G. Fan, H. Zhu, K. Wang, J. Wei, X. Li, Q. Shu, N. Guo, and D. Wu, Graphene/Silicon Nanowire Schottky Junction for Enhanced Light Harvesting, ACS Appl. Mater. Interfaces 3, 721 (2011).

[8] C. C. Chen, M. Aykol, C. C. Chang, A. F. J. Levi, and S. B. Cronin, Graphene-Silicon Schottky Diodes, Nano Lett. 11, 1863 (2011).

[9] K. Chung, C. Lee, and G. Yi, Transferable GaN Layers Grown on ZnO-Coated Graphene Layers for Optoelectronic Devices, Science 330, 655 (2010).

[10] Y. M. Lin, K. A. Jenkins, A. Valdes-Garcia, J. P. Small, D. B. Farmer, and P. Avouris, Operation of Graphene Transistors at Gigahertz. Frequencies Nano Lett. 9, 422 (2009).

[11] X. Li, C. W. Magnuson, A. Venugopal, J. An, J. W. Suk, B. Han, M. Borysiak, W. Cai, A. Velamakanni, Y. Zhu et al., Graphene Films with Large Domain Size by a Two-Step Chemical Vapor Deposition Process, Nano Lett. 10, 4328 (2010).

[12] S. Y. Han, J. Y. Shin, B. T. Lee, and J.L. Lee, Microstructural Interpretation of $\mathrm{Ni}$ Ohmic Contact on n-Type 4H-SiC, J. Vac. Sci. Technol., B 20, 1496 (2002).

[13] S. M. Sze, Physics of Semiconductor Devices (John Wiley, New York, 1981), 2nd ed.

[14] P. H. Hao, L. C. Wang, F. Deng, S. S. Lau, and J. Y. Cheng, On the Low Resistance Au/Ge/Pd Ohmic Contact to n-GaAs, J. Appl. Phys. 79, 4211 (1996).

[15] S. Ruvimov, Z. L. Weber, J. Washburn, K. J. Duxstad, E. E. Haller, Z.-F. Fan, S. N. Mohammad, W. Kim, A.E. Botchkarev, and H. Morkoç, Microstructure of Ti/Al and Ti/Al/Ni/Au Ohmic Contacts for $n-G a N$, Appl. Phys. Lett. 69, 1556 (1996).

[16] H. Cao, Q. Yu, L. A. Jauregui, J. Tian, and W. Wu, Electronic Transport in Chemical Vapor Deposited Graphene Synthesized on Cu: Quantum Hall Effect and Weak Localization, Appl. Phys. Lett. 96, 122106 (2010).

[17] A. Das, S. Pisana, B. Chakraborty, S. Piscanec, S. K. Saha, U. V. Waghmare, K. S. Novoselov, H. R. Krishnamurthy, A. K. Geim, A.C. Ferrari, and A. K. Sood, Monitoring Dopants by Raman Scattering in an Electrochemically Top-gated Graphene Transistor, Nature Nanotech. 3, 210 (2008).

[18] S. Tongay, K. Berke, M. Lemaitre, Z. Nasrollahi, D. B. Tanner, A. F. Hebard, and B. R. Appleton, Stable Hole Doping of Graphene for Low Electrical Resistance and 
High Optical Transparency, Nanotechnology 22, 425701 (2011).

[19] C.-Y. Su, D. Fu, A.-Y. Lu, K.-K. Liu, Y. Xu, Z.-Y. Juang, and L.-J. Li, Transfer Printing of Graphene Strip from the Graphene Grown on Copper Wires, Nanotechnology 22, 185309 (2011).

[20] R. T. Tung, Recent Advances in Schottky Barrier Concepts, Mater. Sci. Eng. R 35, 1 (2001).

[21] Y. J. Yu, Y. Zhao, S. Ryu, L.E. Brus, K. S. Kim, and P. Kim, Tuning the Graphene Work Function by Electric Field Effect, Nano Lett. 9, 3430 (2009).
[22] R. T. Tung, Formation of an Electric Dipole at MetalSemiconductor Interfaces, Phys. Rev. B 64, 205310 (2001).

[23] S. Tongay, T. Schumann, and A. F. Hebard, Graphite Based Schottky Diodes Formed on Si, GaAs, and 4H-SiC Substrates, Appl. Phys. Lett. 95, 222103 (2009).

[24] S. Tongay, T. Schumann, X. Miao, B. R. Appleton, and A. F. Hebard, Tuning Schottky Diodes at the Many-LayerGraphene/Semiconductor Interface by Doping, Carbon 49, 2033 (2011). 